better focus injury prevention efforts and make the most impact on specific populations.

Significance This study adds to the field of injury prevention by providing a possible population to target injury prevention efforts and to ultimately, improve patient outcomes.

\section{THE CHARACTERISTICS OF DRUG-IMPAIRED DRIVERS IN THE UNITED STATES}

Toni Rudisill. Virginia University

10.1136/injuryprev-2020-savir.99

Statement of Purpose Driving under the influence of drugs (DUID) is a burgeoning public health concern in the United States. Most studies have focused on the presence of drugs and/or alcohol among fatally injured drivers. Because little is known about individuals who actively drive drug-impaired, the purpose of this study was characterize the demographics and behaviors of these individuals.

Methods/Approach Data from the 2015-2017 National Survey of Drug Use and Health were analyzed. The study population was limited to drivers $\geq 18$ years of age. The outcome was those who self-reported DUID in the past 12 months. Demographic and behavioral characteristics of those who drove drug-impaired vs. not drug impaired were compared via frequencies, percentages, binary and multivariable logistic regression analyses, which accounted for the survey design.

Results Among eligible respondents $(\mathrm{N}=118,741), \quad 4.7 \%$ reported DUID (unweighted). DUID was highest among 18-25 year olds (9\%), unmarried individuals (8\%), non-heterosexuals (10\%), those whom abused or were drug dependent (40\%), engaged in risky behaviors (15\%), and ever received treatment for substance use (15\%). Those who were dependent on or abused marijuana (32\%), heroin (53\%), or methamphetamine $(52 \%)$ reported DUID most frequently. After adjustment for key demographic and behavioral variables, males [odds ratio, $\mathrm{OR}=1.56 ; 95 \%$ confidence interval (CI) 1.4, 1.7], those who abused or were dependent on drugs $(\mathrm{OR}=4.3 ; 95 \%$ CI 3.8 , $4.8)$, exhibited the most risky behaviors $(\mathrm{OR}=3.7 ; 95 \% \mathrm{CI}$ $3.0,4.6)$, non-heterosexuals $(\mathrm{OR}=1.3 ; 95 \% \mathrm{CI} 1.2,1.5)$, and employed individuals $(\mathrm{OR}=1.3 ; 95 \% \mathrm{CI}=1.2,1.5)$ were more likely to engage in DUID.

Conclusions DUID was particularly common among some population sub-groups including young males, non-heterosexuals, employed individuals, and those who abused or were dependent on drugs. These groups may benefit from interventional measures.

Significance and Contribution to Injury and Prevention Science These findings may help direct future interventional efforts to mitigate DUID. Center.

\section{METHODOLOGICAL REVIEW OF STUDIES THAT INVESTIGATED FACTORS ASSOCIATED WITH TRAUMA RECIDIVISM}

Christopher St Vil. University at Buffalo School of Social Work

10.1136/injuryprev-2020-savir.100

Statement of Purpose Trauma recidivists are patients who present to an emergency room or trauma center on more than one occasion for different incidents of traumatic injury.
Although a major public health issue, there has not yet been a systemic review of the literature that synthesizes our understanding of correlates of trauma recidivism. The purpose of this presentation is to report on the findings of a systematic review of the trauma recidivism literature. This oral presentation focuses on the methodological findings and recommendations that resulted from the review.

Methods/Approach 13 data bases were searched from inception to 2017 for studies that explicitly compared hospitalbased trauma recidivists to non-recidivists. The search was conducted using PRISMA guidelines for systematic reviews.

Results Screening resulted in the selection of 29 studies that met the inclusion criteria.

Conclusions The literature review generated findings that could inform the quality of research among victims of violent injury. Methodological considerations to consider include, a) identifying a common 'recidivism window' that allows detection of patients who are at risk for recidivism, b) disaggregate patients by epochs informed by the human development literature, and c) improving on mixed method methodologies.

Significance and Contributions to Injury and Violence Prevention Science Social science concepts and methodology coupled with medical/victim research can help provide a deeper understanding of the meanings and processes that shape both health research and the behaviors of patients that lead to subsequent re-injury. Insights from this research and the collaborations that stem from it can guide the design of health care delivery practices and interventions that acknowledge and adapt to social, cultural, and economic barriers; harness social mechanisms to increase their effectiveness; or even attempt to manipulate social and cultural determinants of health directly.

\section{CORRELATES OF TRAUMA RECIDIVISM: RESULTS FROM A SYSTEMATIC LITERATURE REVIEW}

Christopher St Vil. University at Buffalo School of Social Work

\subsection{6/injuryprev-2020-savir.101}

Statement of Purpose Trauma recidivists are patients who present to an emergency room or trauma center on more than one occasion for different incidents of traumatic injury. The purpose of this presentation is to report on the findings of a systematic review of the trauma recidivism literature with the purpose of identifying correlates for future research aimed at preventing trauma recidivism. This presentation will discuss the factors traditionally explored in trauma recidivism research, which of those factors are deemed critical based on previous research and provide recommendations for future research with this vulnerable population.

Methods/Approach 13 data bases were searched from inception to 2017 for studies that explicitly compared hospitalbased trauma recidivists to non-recidivists. The search was conducted using PRISMA guidelines for systematic reviews.

Results Screening resulted in the selection of 29 studies that met the inclusion criteria.

Conclusions The literature review identified variables across 6 categories that were included in trauma recidivism literature: 1) Demographic, 2) injury related variables, 3) health related variables, 4) substance use, 5) crime/weapons and 6) vehicular causes. Variables within each category are discussed followed by recommendations of variables to include in future research. 